\title{
Impact of activating transcription factor 4 signaling on lipogenesis in HepG2 cells
}

\author{
LU-PING REN ${ }^{1 *}$, XIAN YU $^{1 *}$, GUANG-YAO SONG ${ }^{1}$, PU ZHANG ${ }^{1,2}$, LI-NA SUN $^{1,2}$, \\ SHU-CHUN CHEN ${ }^{1}$, ZHI-JUAN HU ${ }^{3}$ and XUE-MEI ZHANG ${ }^{1}$ \\ ${ }^{1}$ Department of Endocrinology and Metabolism, Hebei General Hospital, Shijiazhuang, Hebei 050051; \\ ${ }^{2}$ Department of Internal Medicine, Hebei Medical University, Shijiazhuang, Hebei 050017; \\ ${ }^{3}$ Department of Nephrology, Hebei General Hospital, Shijiazhuang, Hebei 050051, P.R. China
}

Received May 31,2015; Accepted April 8, 2016

DOI: $10.3892 / \mathrm{mmr} .2016 .5453$

\begin{abstract}
Non-alcoholic fatty liver disease (NAFLD) is a rapidly growing health threat that has previously been associated with lipogenesis. The direct effect of endoplasmic reticulum stress (ERS) inhibition on the induction of lipogenesis has not been investigated in hepatocytes in vitro. The impact of activating transcription factor-4 (ATF4) on the lipogenic pathway and hepatic insulin transduction in liver cells also requires further investigation. In the present study, the triglyceride (TG) content of HepG2 cells stimulated with fructose was investigated using a commercially available enzymatic assay, and the expression levels of lipogenesis-associated factors were determined by western blotting and reverse transcription-quantitative polymerase chain reaction. Notably, the TG content of HepG2 cells was increased following incubation with fructose, which was accompanied by ERS. 4-Phenylbutyric acid, an inhibitor of ERS, lowered the TG content by reducing the mRNA expression levels of sterol regulatory element-binding protein 1 (SREBP-1c) and carbohydrate-responsive element-binding protein (ChREBP), and the protein expression levels of fatty acid synthase (FAS), acetyl-CoA carboxylase (ACC) and stearoyl-CoA desaturase-1 (SCD-1). Conversely, tunicamycin, which is an inducer of ERS, increased the TG content and stimulated the expression of the above lipogeneic markers. ATF4 deficiency relieved TG accumulation and decreased the mRNA expression levels of SREBP-1c and ChREBP, and protein expression levels of FAS, ACC and SCD-1 in fructose-treated HepG2 cells. Conversely, ATF4 overexpression increased the TG content
\end{abstract}

Correspondence to: Professor Guang-Yao Song, Department of Endocrinology and Metabolism, Hebei General Hospital, 348 Heping West Road, Shijiazhuang, Hebei 050051, P.R. China E-mail: sguangyao2@163.com

*Contributed equally

Key words: endoplasmic reticulum stress, fatty liver, lipogenesis, activating transcription factor- 4 by upregulating the mRNA expression levels of SREBP-1c and ChREBP and protein expression levels of FAS, ACC and SCD-1. Inhibition of ERS was shown to protect HepG2 cells against fructose-induced TG accumulation, whereas induction of ERS stimulated hepatic lipogenesis. As a downstream transcription factor of the unfolded protein response, a deficiency in ATF4 attenuates fructose-induced lipogenesis; while an overexpression of ATF4 can induce TG accumulation through stimulating hepatic lipogenesis. The results of the present study suggested that ATF4 may exert various physiological roles in lipid metabolism depending on the nutrient composition. In addition, these results suggested that ATF4 has a role in regulating lipogenesis and in the development of NAFLD; thus ATF4 may be considered a therapeutic target for NAFLD.

\section{Introduction}

Non-alcoholic fatty liver disease (NAFLD) is a rapidly growing epidemic, which is closely associated with insulin resistance and type 2 diabetes (1-3). Developing novel therapeutic interventions requires a comprehensive understanding of the mechanisms by which excess hepatic lipid develops and causes hepatic insulin resistance. Hepatic steatosis is the basic pathophysiological change existing throughout the development of NAFLD and increased de novo lipogenesis has been shown to be a mechanism by which fatty liver develops $(4,5)$. In addition, it has been increasingly recognized that endoplasmic reticulum stress (ERS) is important in the regulation of lipid metabolism in hepatocytes (6-8). Recent studies using genetic or dietary models of fatty liver have indicated a key association between hepatic steatosis and ER stress, as well as the physiological role of the unfolded protein response (UPR) sensors in lipid homeostasis $(5,6,9,10)$.

UPR is a response to the accumulation of unfolded proteins in the ER, characterized by the activation of three distinct signal transduction pathways mediated by inositol-requiring enzyme 1 (IRE1), PKR-like ER kinase (PERK) and activating transcription factor (ATF) $6 \alpha$. ERS occurs in hepatocytes during liver steatosis $(6,11)$. Intervention with ERS inhibitors, including 4-phenylbutyric acid (4-PBA) and tauroursodeoxycholic (TUDCA), demonstrated that resolved ERS can improve hepatosteatosis in ob/ob mice and 4-PBA can reduce 
hepatic lipid accumulation in ob/ob mice by inhibiting ERS (6). In addition, in a fatty liver model, the inhibition of ERS by 4-PBA can attenuate the lipogenic pathway in high-fructose-fed mice (12). However, the direct effect of ERS inhibition and induction on lipogenesis has not been investigated in hepatocytes in vitro.

Furthermore, it has been indicated that two UPR pathways, PERK-eukaryotic initiation factor $2 \alpha$ (eIF2 $\alpha$ )-ATF4 and IRE-1-X-box binding protein 1 (XBP1) pathways, are involved in the stimulation of de novo lipogenesis in the liver (13). The PERK-eIF-2 $\alpha$-ATF4 pathway, which activates transcription factor-4 (ATF4), is reported to be involved in the regulation of lipogenesis (14). ATF4 is the downstream transcription factor along this UPR arm. ATF4 is found to be involved in a number of different physiological events, such as long-term memory (15), osteoblast differentiation (10) glucose and glycogen bisosynthesis (16), and redox homoeostasis (17). Recently, ATF4 has been shown to be important in the regulation of lipid metabolism. ATF4-null mice do not develop NAFLD when induced by a high-fat diet (16). ATF4 deficiency protects mice from high-carbohydrate diet-induced liver steatosis (18). Furthermore, ATF4 deficiency prevents the development of steatosis and hypertriglyceridemia in response to high fructose intake in mice (19). Nevertheless, the direct effect of ATF4 on the lipogenic pathway and hepatic insulin transduction in liver cells requires further investigation.

Therefore, for further understanding of the association between ATF4 and hepatic lipogeneis, the direct effect of ERS on hepatic lipogenesis was investigated in HepG2 cells in the present study. Subsequently, the impact of ATF4 deficiency or overexpression on the hepatic lipogeneic pathway and insulin signaling transduction in HepG2 cells was observed.

\section{Materials and methods}

Cell line and groups. Briefly, HepG2 cells were cultured in minimal essential medium (HyClone, Thermo Fisher Scientific, Inc., Waltham, MA, USA), supplemented with 10\% (v/v) fetal bovine serum (FBS, Sijiqing Tianhang Biotechnology Co. Hangzhou China), $1 \%$ (v/v) nonessential amino acids, $100 \mathrm{U} / \mathrm{ml}$ penicillin and $100 \mu \mathrm{g} / \mathrm{ml}$ streptomycin (Shijiazhuang, China), in a $5 \% \mathrm{CO}_{2}$-humidified atmosphere. Subsequently, HepG2 cells were stimulated with $20 \mathrm{mmol} / \mathrm{l}$ fructose, fructose plus inhibitor 4-PBA (10 or $20 \mathrm{mmol} / \mathrm{l}$; Sigma-Aldrich, St. Louis, MO, USA) or inducer tunicamycin $(0.1,0.5,2$ and $5 \mu \mathrm{g} / \mathrm{ml}$; Abcam, Cambridge, MA, USA).

Transient plasmid transfection. HepG2 cells that had reached $80 \%$ confluence were transfected with ATF4 vector (EX-F0119-M13-5; GeneCopoeia, Inc., Rockville, MD, USA) or AFT4 small interfering (si)RNA (Santa Cruz Biotechnology Inc., Santa Cruz, CA, USA) for upregulation and downregulation of ATF4, respectively, using Lipofectamine 2000 in $2 \mathrm{ml}$ serum-free Minimum Essential Media (MEM; GE Healthcare Life Sciences, Logan, UT, USA). The vacant EX-EGFP-M13 vector (GeneCopoeia, Inc.) and non-transfected cells were used as controls. At $8 \mathrm{~h}$ after transfection the medium was replaced by normal MEM with $10 \%$ FBS for 24 h. Finally, the cells were cultured for $48 \mathrm{~h}$ prior to analysis.
Quantitative detection of triglyceride (TG) in HepG2 cells. To determine the effect of ATF4 on lipid metabolism in HepG2 cells, the cells were washed twice with phosphate-buffered saline (PBS), and lysed on ice with radioimmunoprecipitation buffer (Pulilai Bioengineering Institute, Changchun, China) for $30 \mathrm{~min}$. After centrifugation at $447.2 \mathrm{x} \mathrm{g}$ for $20 \mathrm{~min}$ at $4^{\circ} \mathrm{C}$, the supernatant was transferred to a new tube. The protein concentration was determined by the bicinchoninic acid assay method. TG levels were measured based on an enzymatic assay from Pulilai Bioengineering Institute, according to the manufacturer's instructions.

Oil red $O$ staining. Cultured cells were fixed for $30 \mathrm{~min}$ in $4 \%$ paraformaldehyde (in PBS), and stained for $2 \mathrm{~h}$ with $1 \%$ Oil Red O. The stained sections were imaged with an Olympus microscope and examined in a blinded manner by the pathologist.

Reverse transcription-quantitative polymerase chain reaction $(R T-q P C R)$. Total RNA was extracted from HepG2 cells using TRIzol reagent (Invitrogen; Thermo Fisher Scientific, Inc.), according to the manufacturer's protocol. RNA purity was confirmed by measuring the ratio of absorbance at 260 and $280 \mathrm{~nm}$ on a spectrophotometer. Reverse transcription of RNA $(8 \mu \mathrm{l})$ was conducted according to the instructions of the Easy Script First-Strand cDNA Synthesis Super Mix kit (TransGen Biotech, Co., Ltd., Beijing, China). Specific primers (Table I) for the amplification of regulator sterol regulatory element-binding proteins (SREBP-1c), carbohydrate response element-binding protein (ChREBP) and glyceraldehyde 3-phosphate dehydrogenase (GAPDH) were verified by NCBI BLAST (http://blast.ncbi.nlm.nih.gov/Blast.cgi). qPCR was performed on an ABI PRISM 7300 PCR system (Applied Biosystems, Thermo Fisher Scientific Inc.) using SYBR Green I GoTaq qPCR Master mix (Promega Corporation, Madison, WI, USA). PCR was conducted in a total volume of $25 \mu \mathrm{l}$ with the following reaction conditions: 1 cycle at $95^{\circ} \mathrm{C}$ for $5 \mathrm{~min}$, followed by 40 cycles of $95^{\circ} \mathrm{C}$ for $15 \mathrm{sec}, 58^{\circ} \mathrm{C}$ for $20 \mathrm{sec}$ and $72^{\circ} \mathrm{C}$ for $30 \mathrm{sec}$. The gene expression from each sample was analyzed in duplicate and normalized against GAPDH. The results are expressed as relative gene expression using the $2^{-\Delta \Delta \mathrm{Cq}}$ method (20).

Evaluation of insulin signal transduction. To observe the effect of ATF4 on insulin sensitivity in fructose-treated HepG2 cells, HepG2 cells transfected with specific ATF-4 vector or siRNA were incubated in the presence or absence of $100 \mathrm{nmol} / 1$ insulin for $30 \mathrm{~min}$. The protein expression levels of phosphorylated (p)-Akt/total-Akt and p-glycogen synthase kinase $3 \beta(\mathrm{GSK} 3 \beta) / \mathrm{t}-\mathrm{GSK} 3 \beta$ were then detected by western blotting.

Western blotting. Cytoplasmic and nuclear proteins were extracted from HepG2 cells using $1 \mathrm{ml}$ radioimmunoprecipitation assay buffer (Beijing Dingguo Biotechnology, Co., Ltd., Beijing, China) containing $10 \mu \mathrm{l}$ phenylmethylsulfonyl. The protein concentration was determined using the bicinchoninic acid assay method. The extracted protein samples were mixed with sodium dodecyl sulfate (SDS) loading buffer and boiled for $10 \mathrm{~min}$ prior to loading. Subsequently, protein samples 
Table I. Primer sequences for amplification of SREBP-1c, ChREBP and GAPDH.

\begin{tabular}{lll}
\hline Gene & \multicolumn{1}{c}{ Forward } & \multicolumn{1}{c}{ Reverse } \\
\hline GAPDH & 5'GGATGATGTTCTGGAGAGCC3' & 5'CATCACCATCTTCCAGGAGC3' \\
SREBP-1c & 5'CTTCCGCCCTTGAGCTG3' & 5'CTGGTGTGTCCGTGTGG3' \\
ChREBP & 5'TGCGGGATGAGATTGAGGA3' & 5'TCCAGTTGTGCAGCGTAC3'
\end{tabular}

GAPDH, glyceraldehyde 3-phosphate dehydrogenase; SREBP-1c, sterol regulatory element-binding protein 1; ChREBP, carbohydrate-responsive element-binding protein.

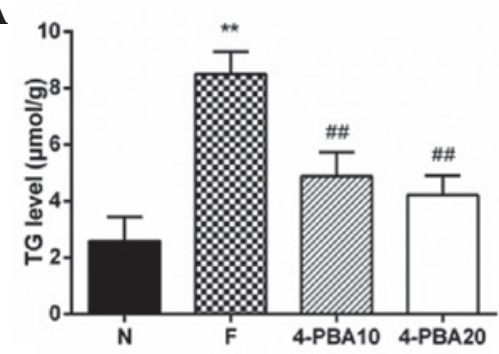

C

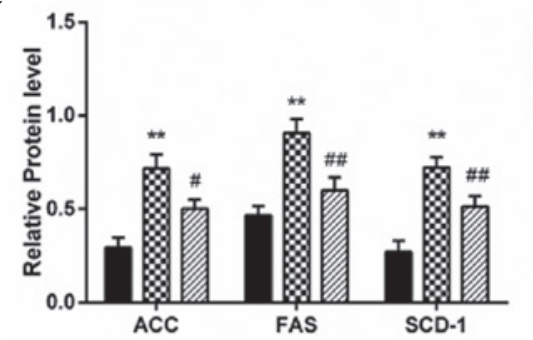

D

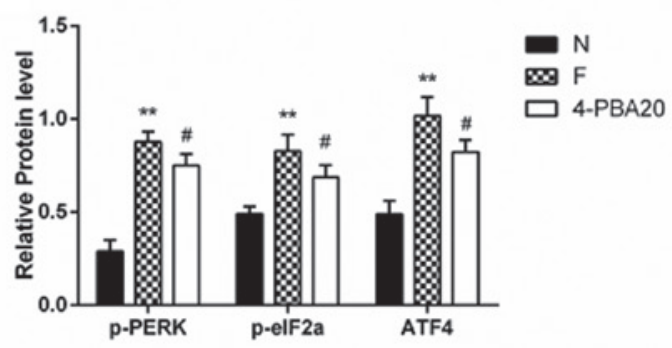

$\mathbf{E}$

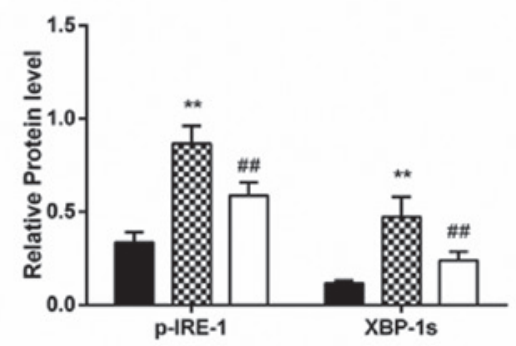

B

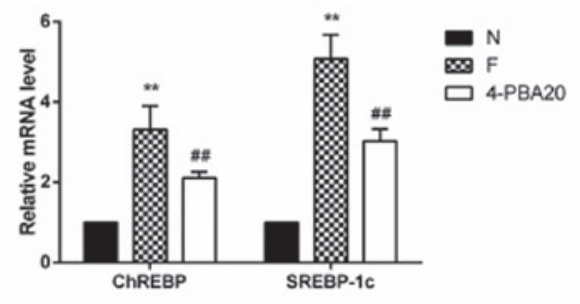

$\infty \mathrm{N}$

4-PBA20
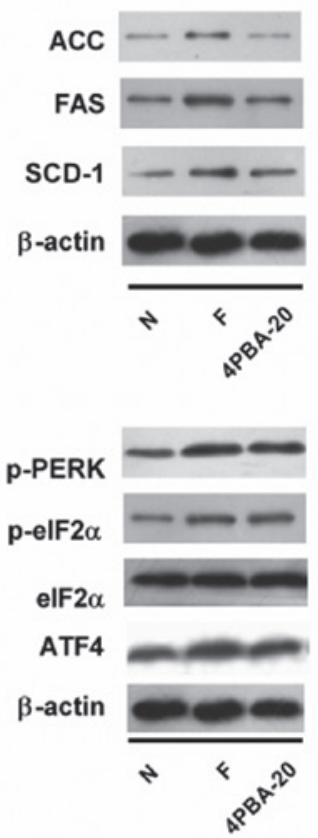

$\mathrm{N}$
$\mathrm{F}$
$4-\mathrm{PBA20}$

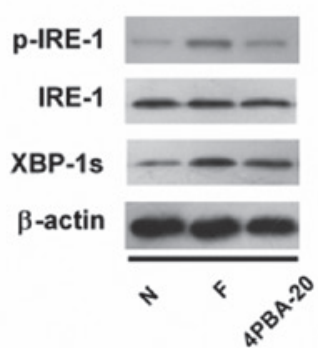

Figure 1. Lipogenesis and ERS were stimulated in fructose-incubated HepG2 cells and were inhibited by 4-PBA. (A) TG was detected after HepG2 cells were incubated in normal medium ( $\mathrm{N}$ group), high fructose $(20 \mathrm{mM})$ (F group) for $72 \mathrm{~h}$, and high fructose following pretreatment for $8 \mathrm{~h}$ with 10 or $20 \mathrm{mM} 4-\mathrm{PBA}$ (4PBA10 and 4PBA20 groups, respectively). (B) mRNA levels of SREBP-1c and ChREBP mRNA were tested by polymerase chain reaction. Fold induction represents relative expression compared with that of the control group. (C) Protein expression of key enzymes (SCD-1, FAS, ACC) in the lipogenic pathway in HepG2 cells were tested by western blotting. $\beta$-actin served as the loading control. (D) Protein expression levels of p-PERK, p-eIF-2 $\alpha /$ IF-2 $\alpha$, ATF4 and CHOP in HepG2 cells. (E) Protein expression levels of $\mathrm{p}-\mathrm{IRE}-1 / \mathrm{IRE}-1$ and XBP-1s in HepG2 cells. Results are presented as the mean \pm standard deviation. ${ }^{*} \mathrm{P}<0.05$ and ${ }^{* *} \mathrm{P}<0.01$ vs. the $\mathrm{N}$ group, and ${ }^{\#} \mathrm{P}<0.05$ and ${ }^{\# \#} \mathrm{P}<0.01$ vs. the F group. ERS, endoplasmic reticulum stress; 4-PBA, 4-phenylbutyric acid; TG, triglyceride; SREBP-1c, sterol regulatory element-binding protein 1; ChREBP, carbohydrate-responsive element-binding protein; SCD-1, stearoyl-CoA desaturase 1; FAS, fatty acid synthase; ACC, acetyl-CoA carboxylase; p-PERK, PKR-like ER kinase; eIF-2 $\alpha$, eukaryotic initiation factor $2 \alpha$; ATF4, activating transcription factor-4; CHOP, C/EBP homologous protein; IRE-1, inositol-requiring enzyme 1; XBP-1s, spliced X-box binding protein 1; p-, phosphorylated. 
A

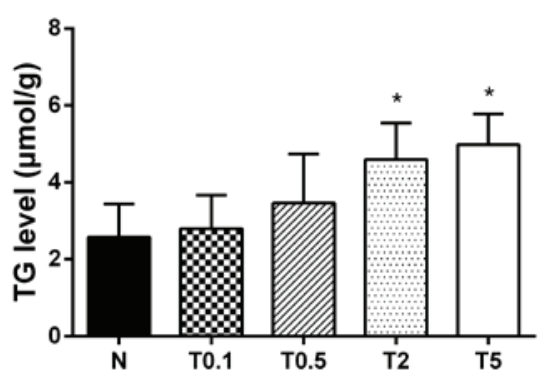

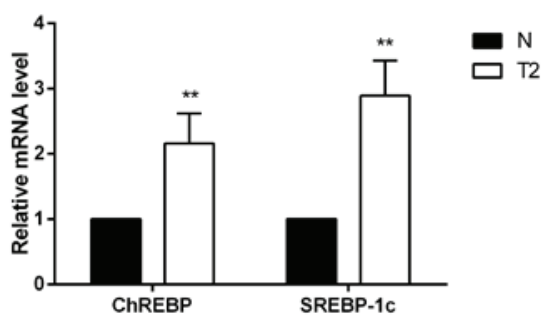

C

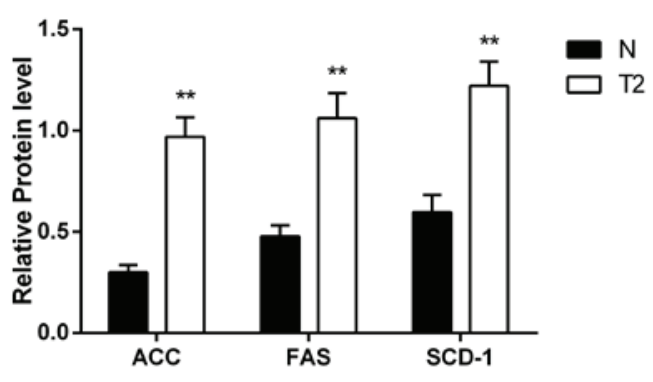

D

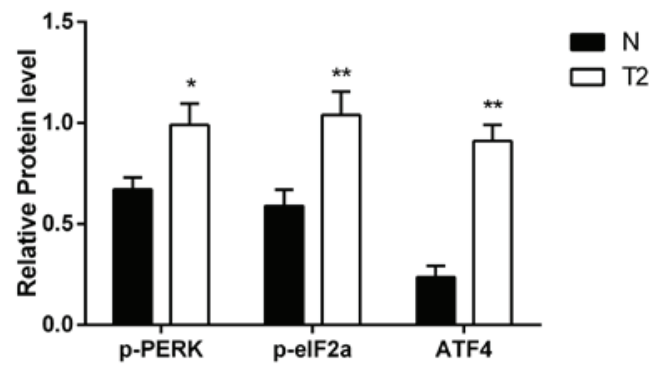

$\mathbf{E}$

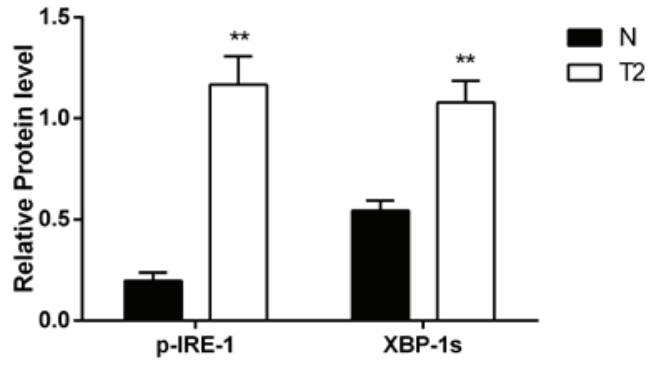

ACC

FAS

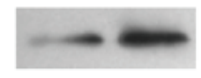

SCD-1

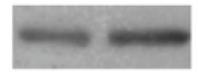

$\beta$-actin

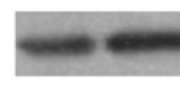

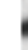
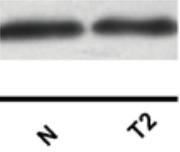

p-PERK

p-elF2 $\alpha$

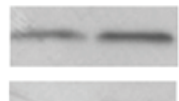

elF2 $\alpha$

ATF4

$\beta$-actin

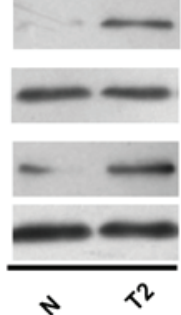

p-IRE-1

IRE-1

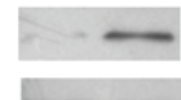

XBP-1s

$\beta$-actin

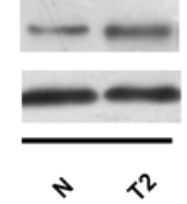

Figure 2. Lipogenesis was stimulated and ERS occurred in HepG2 cells cultured in different concentrations of tunicamycin. (A) TG content in the HepG2 cells exposed to different concentrations $(0.1,0.5,2$ or $5 \mu \mathrm{g} / \mathrm{ml}$; T0.1, T0.5, T2, T5 groups, respectively) of tunicamycin [mean \pm standard deviation (SD), n=3]. (B) SREBP-1c and ChREBP mRNA levels in HepG2 cells were detected by polymerase chain reaction (mean \pm SD, $n=6$ ). (C) Protein expression of critical enzymes (SCD-1, FAS and ACC) in the lipogenic pathway in HepG2 cells incubated with tunicamycin (mean \pm SD, n=3). (D) Protein expression of p-PERK, p-eIF- $2 \alpha$ and ATF4 were increased significantly in HepG2 cells incubated with tunicamycin (mean \pm SD, n=3). (E) Protein expression of p-IRE-1/IRE-1 and XBP-1s were increased significantly in HepG2 cells incubated with tunicamycin (mean $\pm \mathrm{SD}, \mathrm{n}=3$ ). ${ }^{*} \mathrm{P}<0.05$ and ${ }^{* *} \mathrm{P}<0.01$ vs. the $\mathrm{N}$ group. ERS, endoplasmic reticulum stress; TG, triglyceride; SREBP-1c, sterol regulatory element-binding protein 1; ChREBP, carbohydrate-responsive element-binding protein; SCD-1, stearoyl-CoA desaturase 1; FAS, fatty acid synthase; ACC, acetyl-CoA carboxylase; p-PERK, PKR-like ER kinase; eIF-2 $\alpha$, eukaryotic initiation factor $2 \alpha$; ATF4, activating transcription factor-4; CHOP, C/EBP homologous protein; IRE-1, inositol-requiring enzyme 1; XBP-1s, spliced X-box binding protein 1; p-, phosphorylated; $\mathrm{N}$, normal medium group.

were separated by $10 \%$ SDS-PAGE and transferred onto polyvinylidene difluoride membranes (EMD Millipore, Billerica, MA, USA). The membrane was blocked for $2 \mathrm{~h}$ at $37^{\circ} \mathrm{C}$ with non-fat milk or $5 \%$ bovine serum albumin (Ameresco, Inc., Framingham, MA, USA) in Tris-buffered saline containing 0.05\% Tween 20 (TBST). The membrane was then incubated with the following primary antibodies overnight at $4^{\circ} \mathrm{C}$ : Rabbit
anti-stearoyl-CoA desaturase 1 (SCD-1; cat. no. 2438; 1:1,000), acetyl-CoA carboxylase (ACC; cat. no. 3662; 1:800), ATF4 (cat. no. 11815; 1:1,000), C/EBP homologous protein (CHOP; cat. no. 5554; 1:1,000), Akt (cat. no. 9272; 1:1,000), p-Akt (Thr308) (cat. no. 9271; 1:1,000), GSK3 $\beta$ (cat. no. 9331; 1:1,000), p-GSK3 $\beta$ (Ser21/9) (cat. no. 5676; 1:1,000), eIF2- $\alpha$ (cat. no. 9722; 1:1,000) and p-eIF2- $\alpha$ (Ser51) (cat. no. 5199; 1:1,000) 
A

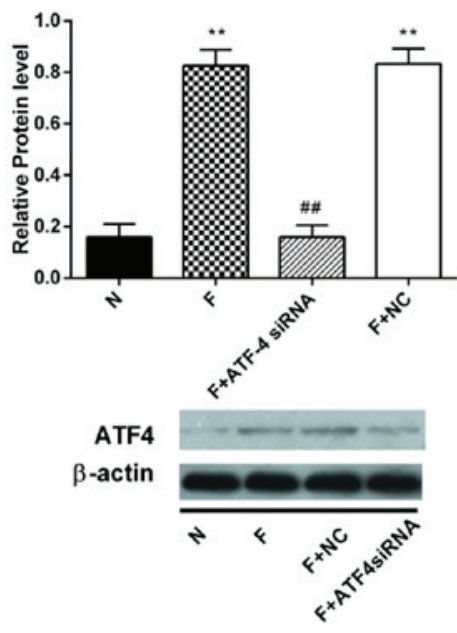

C

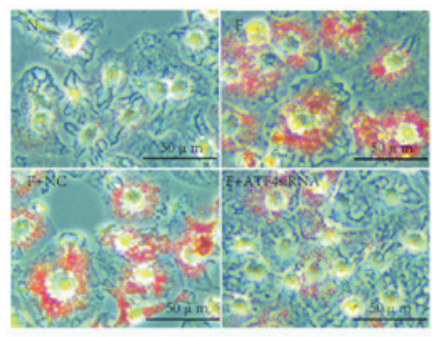

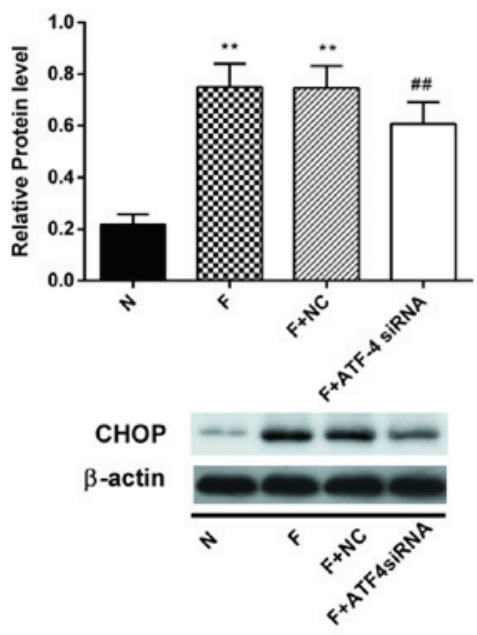

D

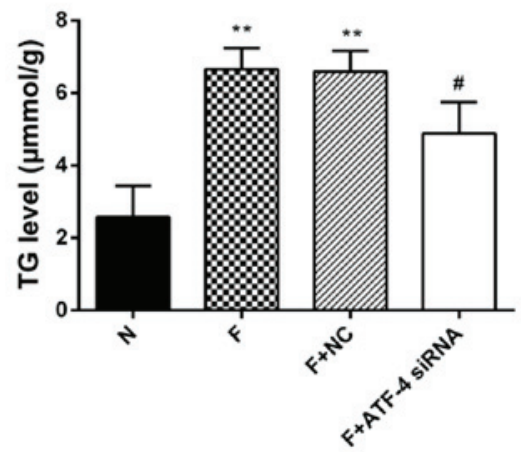

Figure 3. Lipid accumulation was attenuated in fructose-incubated ATF4-deficient HepG2 cells. Protein expression level of (A) ATF4 and (B) CHOP in HepG2 cells after $8 \mathrm{~h}$ of transfection with ATF4 siRNA (mean \pm SD, n=3). (C) Lipid droplets were decreased in ATF4-deficient HepG2 cells cultured with $20 \mathrm{mM}$ fructose as shown by Oil red O staining. (D) TG content was decreased in ATF4-deficient HepG2 cells cultured with $20 \mathrm{mM}$ fructose. ${ }^{* *} \mathrm{P}<0.01$, vs. the $\mathrm{N}$ group, ${ }^{\#} \mathrm{P}<0.05$ and ${ }^{\# \#} \mathrm{P}<0.01$ vs. the $\mathrm{F}$ group. ATF4, activating transcription factor-4; CHOP, C/EBP homologous protein; siRNA, small interfering RNA; TG, triglyceride; N, normal medium group; F, fructose group; NC, fructose + transfection reagents group; siRNA, small interfering RNA.

polyclonal antibodies, and rabbit anti-XBP-1 monoclonal antibody (cat. no. 12782; 1:1,000), from Cell Signaling Technology Inc. (Beverly, MA, USA); rabbit anti-PERK (cat. no. sc-13073; 1:500), p-PERK (Thr980) (cat. no. sc-32577; 1:500) and fatty acid synthase (FAS; cat. no. C-20; 1:500) polyclonal antibodies from Santa Cruz Biotechnology Inc.; rabbit anti-IRE-1 (cat. no. ab62570; 1:1,000) and p-IRE-1 (Ser724) (cat. no. ab48187; 1:1,000) polyclonal antibodies from Abcam; and mouse anti- $\beta$-actin monoclonal antibody (cat. no. 66009-1-Ig;1:5,000) from Cambridge Bioscience (Cambridge, UK). Subsequently, the membrane was washed three times with TBST and then incubated with horseradish peroxidase-conjugated goat anti-rabbit (cat. no. L3012-2; 1:5,000) and anti-mouse (L3032-2; 1:5,000) secondary antibodies (Signalway Antibody, College Park, MD, USA) at room temperature for $2 \mathrm{~h}$. After rinsing with TBST three times, the membrane was treated with enhanced chemiluminescence (ECL) solution (Pierce Biotechnology; Thermo Fisher Scientific Inc.) and bands were detected by exposing the blots to X-ray films (Ruike Medical Instrument, Xiamen, China). $\beta$-actin served as an internal control protein. Band intensities were quantified using ImageJ software, version 1.42q (https://imagej.nih.gov/ij/).

Statistical analysis. Data are presented as the mean \pm standard deviation of three independent experiments. Statistical analyses were performed using SPSS 11.5 software (SPSS, Inc., Chicago, IL, USA). Differences among groups were compared by one-way analysis of variance. $\mathrm{P}<0.05$ was considered to indicate a statistically significant difference.

\section{Results}

4-PBA alleviates high fructose-induced lipogenesis through inhibiting ERS in the HepG2 cells. PBA $(20 \mathrm{mM})$ pretreatment inhibited fructose-induced PERK (p-PERK, Thr980) and eIF2 $\alpha$ (p-eIF2 $\alpha$, Ser51) phosphorylation and suppressed ERS-induced lipogenesis. In order to determine the effect of ERS on lipid metabolism, HepG2 cells were pretreated with fructose plus 10, $20 \mathrm{mM}$ of 4-PBA. The TG level was induced in high fructose conditions but ameliorated by 4-PBA intervention $(\mathrm{P}<0.01)$. TG was lower in the $20 \mathrm{mM}$ 4-PBA group than in the $10 \mathrm{mM}$ 4-PBA group, however, the levels were not identified to be significantly different $(\mathrm{P}=0.337$; Fig. 1A).

Incubation with $20 \mathrm{mM}$ 4-PBA attenuates lipogenesis in the liver and protects against high fructose-induced SREBP-1c and ChREBP gene expression (all $\mathrm{P}<0.01$; Fig. 1B). Accordingly, the protein contents of SCD-1, FAS and ACC were decreased in cells treated with 4-PBA (all $\mathrm{P}<0.01$; Fig. 1C). Protein levels of p-PERK, ATF4 and CHOP were increased by fructose incubation but decreased significantly by 4-PBA intervention (all 
A

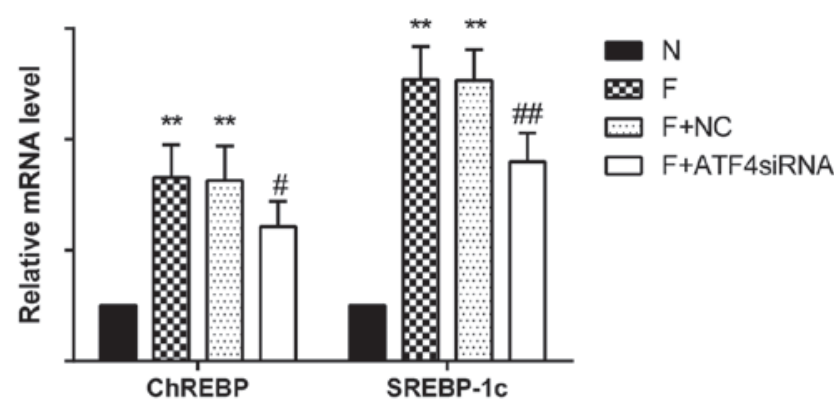

B
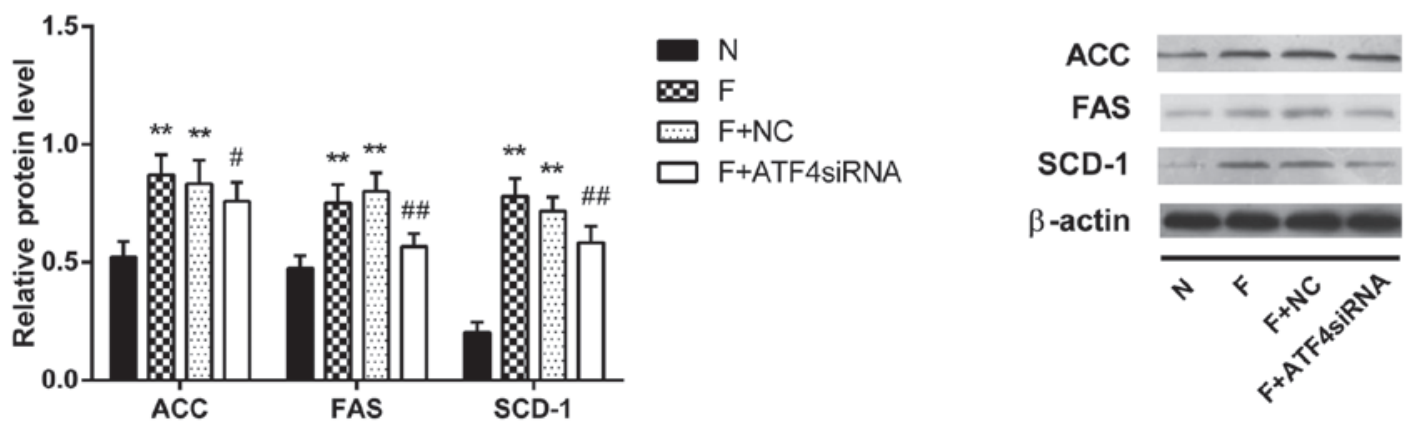

Figure 4. Expression levels of lipogenic markers were downregulated in fructose-incubated ATF4-deficient HepG2 cells. (A) Levels of SREBP-1c, ChREBP mRNA were tested by polymerase chain reaction [mean \pm standard deviation (SD), $\mathrm{n}=6$ ]. (B) Protein expression levels of key enzymes in the lipogenic pathway (SCD-1, FAS and ACC) in ATF4-deficient HepG2 cells were determined by western blotting and densitometry. $\beta$-actin expression served as a loading control. Fold induction represents relative expression level compared with that of the $\mathrm{N}$ group, $($ mean $\pm \mathrm{SD}, \mathrm{n}=3) .{ }^{* *} \mathrm{P}<0.01$ vs. the $\mathrm{N}$ group; ${ }^{\# \mathrm{P}}<0.05$ and ${ }^{\# \#} \mathrm{P}<0.01$ vs. the F group. SREBP-1c, sterol regulatory element-binding protein 1; ChREBP, carbohydrate-responsive element-binding protein; SCD-1, stearoyl-CoA desaturase 1; FAS, fatty acid synthase; ACC, acetyl-CoA carboxylase; ATF4, activating transcription factor-4; siRNA, small interfering RNA; N, normal medium group; $\mathrm{F}$, fructose group; $\mathrm{F}+\mathrm{NC}$, fructose + transfection reagents group.

$\mathrm{P}<0.05)$. The ratios of $\mathrm{p}-\mathrm{eIF}-2 \alpha / \mathrm{eIF}-2 \alpha$ and $\mathrm{p}-\mathrm{IRE}-1 / \mathrm{IRE}-1$ were significantly increased by fructose incubation $(\mathrm{P}<0.05)$ and they were markedly decreased after 4-PBA intervention, although without statistical significance ( $P>0.05$; Fig. 1D and E).

Tunicamycin induces lipogenesis via stimulation of ERS. Compared with HepG2 cells cultured in normal medium (N group), HepG2 cells treated with various concentrations of tunicamycin $(0.1,0.5,2$ or $5 \mu \mathrm{g} / \mathrm{ml}$; T0.1, T0.5, T2, T5 groups, respectively) showed increased TG levels in a concentration-dependent manner (Fig. 2A). TG levels in the T2 and T5 groups were significantly increased compared with that in the $\mathrm{N}$ group. As incubation with $5 \mu \mathrm{g} / \mathrm{ml}$ tunicamycin induced apoptosis to a greater extent than $2 \mu \mathrm{g} / \mathrm{ml}$ tunicamycin, with nearly equal effect of inducing ERS, $2 \mu \mathrm{g} / \mathrm{ml}$ was selected as the tunicamycin intervention concentration for further studies. RT-qPCR results demonstrated a significant increase in the abundance of SREBP-1c and ChREBP mRNA levels in HepG2 cells cultured in tunicamycin-enriched medium compared with control $(\mathrm{P}<0.01$; Fig. $2 \mathrm{~B})$. Key lipogenic enzyme protein expression of critical enzymes (SCD-1, FAS and ACC) in the lipogenic pathway in HepG2 cells were increased compared with the normal group (all $\mathrm{P}<0.01$; Fig. 2C). Tunicamycin inhibits N-linked glycosylation of proteins, leading to high levels of stressors which were expected to rapidly activate two arms of the UPR. Consistent with this, p-PERK, ATF4, p-IRE-1/IRE-1, CHOP protein levels were all significantly increased (all $\mathrm{P}<0.01$ except $\mathrm{P}=0.05$ for $\mathrm{p}$-PERK) (Fig. 2D and $\mathrm{E}$ ).
Silencing of ATF4 expression prevents high fructose-induced lipid deposition in HepG2 cells by inhibiting lipogenesis. ATF4 protein was downregulated after transfection with an siRNA targeting ATF4 (F+ATF4 siRNA group) compared with cells treated with fructose ( $\mathrm{F}$ group) or cells treated with transfection reagents and fructose $(\mathrm{F}+\mathrm{NC}$ group) $(\mathrm{P}<0.01$; Fig. 3A). The CHOP protein content was increased in the $\mathrm{F}$ group and significantly decreased in the F+ATF4 siRNA group $(\mathrm{P}<0.01$; Fig. 3B). Lipid droplets (red-stained granules) were decreased in F+ATF4 siRNA group cells as shown by Oil red O staining (Fig. 3C). Accordingly, TG accumulation was reduced in the F+ATF4 siRNA group compared with the F group $(\mathrm{P}<0.05$; Fig. 3D).

Upstream lipogenic transcriptional factors including ChREBP $(\mathrm{P}<0.05)$ and SREBP-1c mRNA $(\mathrm{P}<0.01)$ were significantly downregulated in ATF4-deficient HepG2 cells (Fig. 4A). Downstream protein levels of key lipogenic enzymes ACC, FAS and SCD-1 ( $\mathrm{P}<0.05, \mathrm{P}<0.01$ and $\mathrm{P}<0.01$, respectively) were significantly downregulated in ATF4-deficient HepG2 cells (Fig. 4B).

Effect of ATF4 overexpression on lipid synthesis in HepG2 cells. An ATF4 plasmid and empty vector as negative control were respectively transfected into HepG2 cells. After $8 \mathrm{~h}$ of transfection, ATF4 protein levels were significantly increased $(\mathrm{P}<0.01$; Fig. 5A). The protein content of $\mathrm{CHOP}$ was shown to be significantly increased in HepG2 cells after transfection with the ATF4 plasmid compared with the untransfected cells (Fig. 5B). Lipid droplets (red-stained granule) were increased 


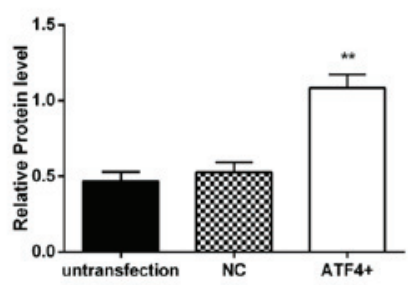

C
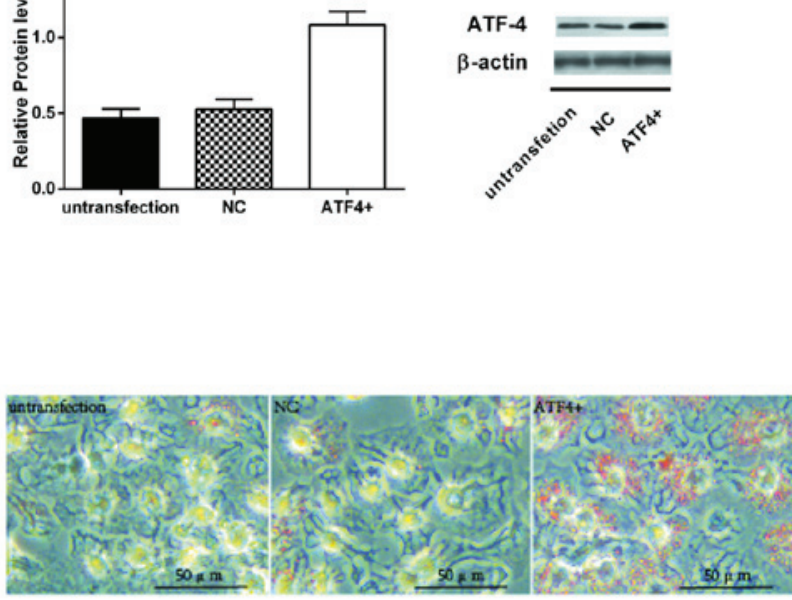

B

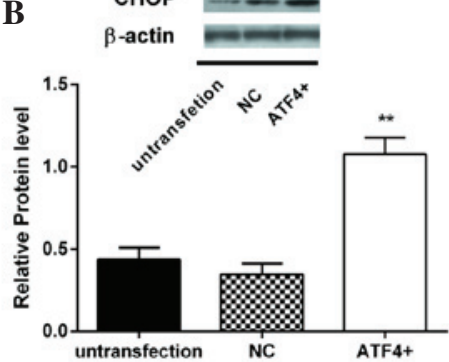

D

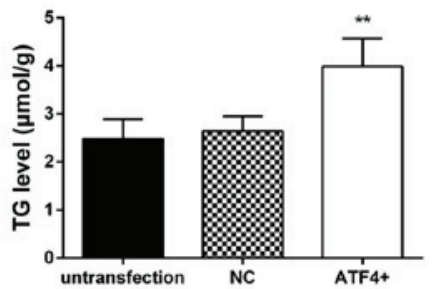

Figure 5. ATF4 overexpression induced TG accumulation in HepG2 cells. (A) Protein expressions level of ATF4 after $8 \mathrm{~h}$ of ATF4 vector transfection. (B) Protein expression of CHOP was increased in ATF4 overexpressed HepG2 cells. (C) Lipid droplets were increased in ATF4-overexpressed HepG2 cells as shown by Oil red O staining. (D) TG content was increased in ATF4 overexpressed HepG2 cells. Data are presented as the mean \pm standard deviation (n=3). ${ }^{* *} \mathrm{P}<0.01$ vs. untransfection group. ATF4, activating transcription factor- 4 ; TG, triglyceride; CHOP, C/EBP homologous protein; NC, fructose + transfection reagents group.

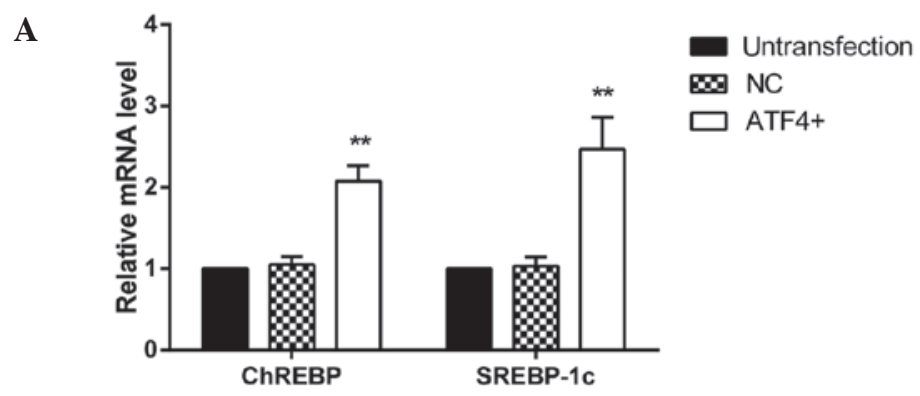

B
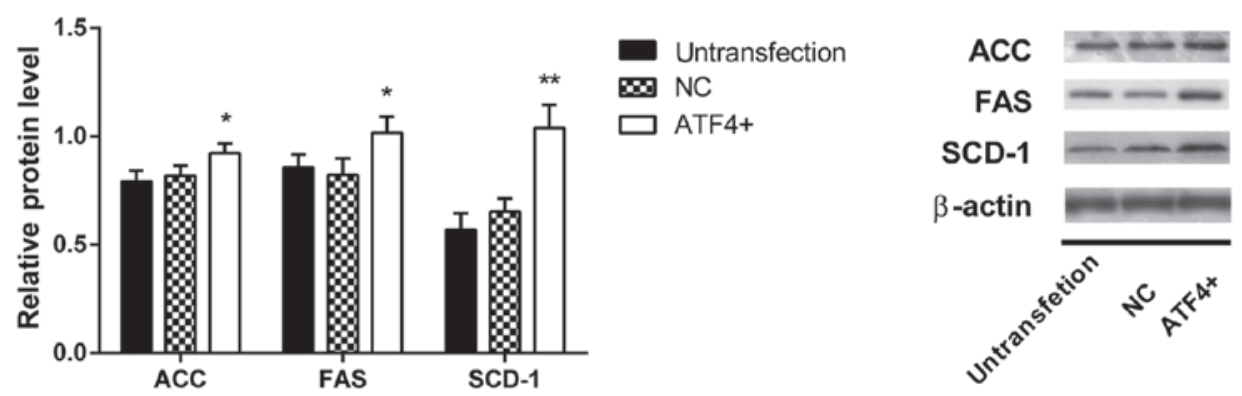

Figure 6. Expression levels of lipogenic markers were upregulated in ATF4-overexpressing HepG2 cells. (A) mRNA levels of SREBP-1c and ChREBP were increased in ATF4-overexpressing HepG2 cells. (B) Protein expression levels of key enzymes in the lipogenic pathway (SCD-1, FAS and ACC) were increased in ATF4-overexpressing HepG2 cells. $\beta$-actin served as a loading control. ${ }^{*} \mathrm{P}<0.05$ and ${ }^{* *} \mathrm{P}<0.01$ vs. the untransfection group. Data are presented as the mean \pm standard deviation. SREBP-1c, sterol regulatory element-binding protein 1; ChREBP, carbohydrate-responsive element-binding protein; SCD-1, stearoyl-CoA desaturase 1; FAS, fatty acid synthase; ACC, acetyl-CoA carboxylase; ATF4, activating transcription factor-4; NC, fructose + transfection reagents group.

in HepG2 cells transfected with ATF4 plasmid (Fig. 5C). In addition, TG content was higher in HepG2 cells transfected with ATF4 plasmid than cells grown in normal medium and cells transfected with empty vector $(\mathrm{P}<0.01$; Fig. 5D).

RT-qPCR results demonstrated a significant increase in the abundance of SREBP-1c mRNA levels in HepG2 cells transfected with the ATF4 plasmid (all $\mathrm{P}<0.05$ ); whereas there was no change in ChREBP mRNA levels (Fig. 6A). Protein contents of ACC, FAS and SCD-1 were significantly increased compared with that in the untransfected cells $(\mathrm{P}<0.05, \mathrm{P}<0.05$ and $\mathrm{P}<0.01$ respectively; Fig. 6B).

Effects of ATF4 silencing and overexpression on insulin sensitivity in HepG2 cells. The decreased sensitivity of HepG2 cells 
A

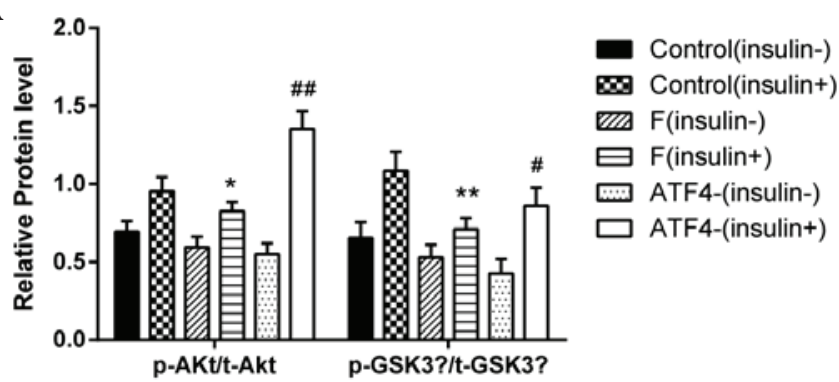

B

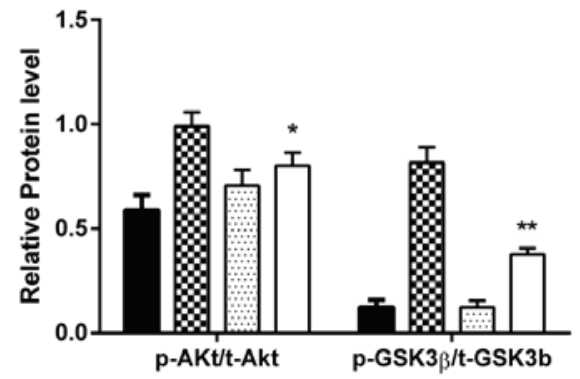

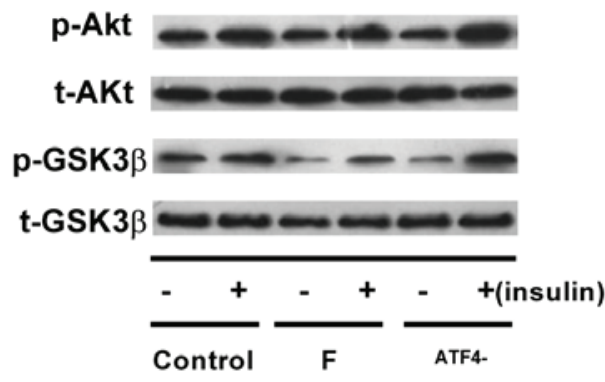

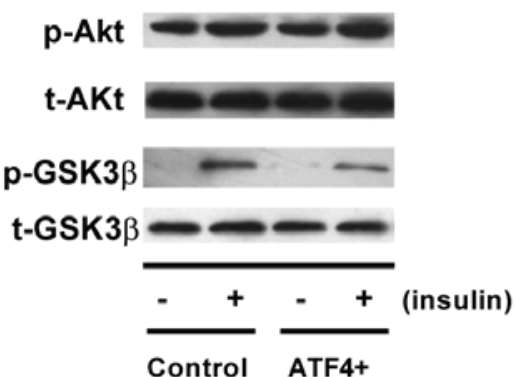

Figure 7. Effects of ATF4 silencing and overexpression on the insulin sensitivity of HepG2 cells. HepG2 cells transfected with ATF4 vector or ATF4 small interfering (si)RNA were incubated in the presence or absence of $100 \mathrm{nmol} / \mathrm{l}$ insulin for $30 \mathrm{~min}$ and the protein expression levels of $\mathrm{p}$-Akt, $\mathrm{t}$-Akt, $\mathrm{p}$-GSK $\beta$ and $\mathrm{t}$-GSK $\beta$ were determined by western blotting. (A) Protein content ratios of $\mathrm{p}$-Akt/t-Akt and p-GSK $\beta / \mathrm{t}-\mathrm{GSK} \beta$ were increased following insulin stimulation of HepG2 cells transfected with ATF4 siRNA, indicating an increased insulin sensitivity. (B) Protein content ratios of p-Akt/t-Akt and p-GSK $\beta / \mathrm{t}-\mathrm{GSK} \beta$ were decreased after insulin stimulation in ATF4-overexpressing HepG2 cells, indicating a decreased insulin sensitivity. Data are presented as the mean \pm standard deviation $(\mathrm{n}=3)$. ${ }^{*} \mathrm{P}<0.05,{ }^{* *} \mathrm{P}<0.01$ vs. the control (insulin + ) group. ${ }^{\#} \mathrm{P}<0.05,{ }^{\# \#} \mathrm{P}<0.01$ vs. the $\mathrm{F}$ (insulin + ) group. GSK $\beta$, glycogen synthase kinase $\beta$; p-, phosphorylated; t-Akt, total-Akt; F, fructose group.

to insulin following stimulation with fructose was improved by ATF4 silencing, as indicated by increased $\mathrm{p}-\mathrm{Akt} / \mathrm{t}-\mathrm{AKt}$ $(\mathrm{P}<0.01)$ and $\mathrm{p}-\mathrm{GSK} / \mathrm{t}-\mathrm{GSK}(\mathrm{P}<0.05)$ ratios following insulin stimulation, as compared with the F group (Fig. 7A). Conversely, ATF4 overexpression decreased the ratios of p-Akt/t-AKt $(\mathrm{P}<0.05)$ and p-GSK/t-GSK $(\mathrm{P}<0.01)$ following insulin stimulation, as compared with the $\mathrm{F}$ group (Fig. 7B).

\section{Discussion}

Increased hepatic lipogenesis is one of the mechanisms underlying the development of NAFLD. Recent studies indicate that ERS is involved in the development of NAFLD and is associated with lipogeneis $(21,22)$. It has been shown that ERS occurs in the fatty livers of genetically obese, chronic high-fat-fed or high-fructose-fed rodents (6,11-12). Resolved hepatic ERS by 4-PBA or tauroursodeoxycholic acid can attenuate hepatosteatosis in ob/ob mice or high-fructose-fed mice $(6,12)$. Nevertheless, the direct impact of ERS inhibition and induction on lipogenesis has not been investigated in hepatocytes in vitro. Therefore, the present study investigated the impact of 4-PBA on lipogenesis in HepG2 cells cultured in a high concentration of fructose, which stimulates lipogenisis in the liver $(23,24)$. The effect of ERS induction by tunicamycin on hepatic lipogenesis was then determined.

This study showed that fructose increased the mRNA expression of SREBP-1c and ChREBP, which are upstream transcription factors of hepatic lipogenesis $(25,26)$. Downstream enzymes including ACC, FAS and SCD-1 were subsequently activated. ERS was observed in fructose-cultured HepG2 cells as reflected by the activation of the PERK-eIF2 $\alpha$-ATF4 and IRE-1-XBP1 pathways. The inhibition of ERS by 4-PBA decreased the gene expression of SREBP-1c and ChREBP, and the protein expression of ACC, FAS and SCD-1. These results are consistent with our previous animal study, which showed that 4-PBA can inhibit de novo lipogenesis in high-fructose-fed mice (12). To further investigate the impact of ERS on hepatic lipogenesis, ERS was induced by tunicamycin in HepG2 cells. Accompanied by the occurrence of ERS, the lipogenesis pathway was shown to be activated. Lipogenesis was stimulated by ERS inducers with subsequent TG accumulation. These findings demonstrated the regulatory role of ERS in lipogenesis in liver cells.

Since it is indicated that ERS is involved in lipogenesis in the liver, several previous studies have investigated the association between ERS and lipogenesis. Studies demonstrated that the UPR exhibits a regulatory effect on lipid synthesis in the liver (27,28). UPR is a self-protective mechanism in the ER to cope with ERS and is regarded as a marker of ERS. A previous study demonstrated that IRE-1-XBP1 and PERK-eIF2 $\alpha$-ATF4 pathways, two UPR pathways, are involved in regulating lipogenesis (5). The IRE1-XBP1 pathway has been linked to adipogenesis in mouse embryonic fibroblasts and 3T3-L1 cells $(29,30)$. Based on an XBP1-deficient mouse model study, $\mathrm{XBP}-1$ has been established as a novel transcription factor governing hepatic lipogenesis (31). It has also been demonstrated that targeted deletion of PERK in mammary epithelium reduced free fatty acids in mouse milk and led to growth retardation in suckling pups (32). PERK deletion resulted in reduced 
expression of several lipogenic genes, including SREBP1 (33). In addition, dephosphorylation of eIF- $2 \alpha$ (translation initiation factor $2 \alpha$ ) enhances glucose tolerance and attenuates hepatosteatosis in mice (13). The present study focused on the impact of ATF4 on lipogenesis in HepG2 cells.

ATF4 is expressed in a wide variety of tissues and is stimulated in response to various cellular stresses, such as amino acid deprivation and integrated stress stimulation $(34,35)$. ATF4 is also the downstream transcriptional factor involved in the PERK-eIF2 $\alpha$-ATF4 pathway in UPR. One study showed that ATF4 null mice have increased energy expenditure and are resistant to diet-induced obesity (16). Further studies indicated that mice with ATF4 protein deficiency are resistant to liver steatosis induced by a high-fat-diet, high carbohydrate diet and high-fructose diet $(16,18,19)$. In the present study, ATF4 deficiency attenuated the TG accumulation induced by fructose-incubated liver cells. Conversely, ATF4 overexpression induced TG accumulation in HepG2 cells, indicating the impact of ATF4 on lipid metabolism in the liver.

Previous studies indicated that ATF4 is involved in the regulation of numerous aspects of lipid metabolism. ATF4-deficient mice exhibited increased expression of genes associated with lipolysis and $\beta$-oxidation in white adipose tissue, including hormone sensitive lipase, carnitine palmitoyltransferase 1 , and medium-chain acyl-CoA dehydrogenase (14). In addition to lipid oxidation and lipolysis, the impact of ATF4 deficiency on de novo lipogenesis in the liver was investigated and it was demonstrated that the gene and protein expression of SREBP-1c, ACC and FAS were reduced in livers of high-fructose-fed ATF $4^{-/}$mice (19). In the present study, the results were consistent with these findings. In HepG2 cells with silenced ATF4 expression, when cultured with a high concentration of fructose, the upstream lipogenic transcription factor SREBP-1c was downregulated compared with that in control cells. In addition, in the present study, it was demonstrated that ChREBP, another upstream lipogenic transcription factor, was also downregulated in fructose-incubated HepG 2 cells accompanied by reduced protein expression of downstream lipogenic enzymes.

The regulatory effect of ATF4 on lipogenesis in HepG2 cells was further demonstrated by the findings in ATF4-overexpressed HepG2 cells. ATF4 overexpression stimulated the gene expression of SREBP-1c and ChREBP. ATF4 overexpression increased protein expression of ACC, FAS and SCD-1. Different from these findings, however, a previous study showed that in $\mathrm{ATF}^{-/}$mice fed on a high-carbohydrate diet, ATF4 deficiency reduced the expression of SCD-1 but did not have an effect on the expression of SREBP-1 and ChREBP (18). These contrasting observations indicate that ATF4 may exhibit different physiological roles in lipid metabolism in the context of different nutrient compositions. Further investigation is required to clarify how ATF4 regulates the expression of SREBP-1c and ChREBP.

In addition to the impact of ATF4 on lipogenesis, the influence of ATF4 on insulin action in liver cells was demonstrated. It was shown that ATF4 overexpression induces insulin resistance in HepG2 cells, while ATF4 deficiency can protect insulin sensitivity in fructose-incubated HepG2 cells. The mechanism by which ATF4 deficiency increases insulin sensitivity may be indirect and result from the inhibition of lipogenesis and corresponding decreases in TG accumulation. Whether ATF4 has a direct regulatory effect on the insulin signaling pathway requires further investigation.

In conclusion, the present study demonstrated that inhibition of ERS protects HepG2 cells against fructose-induced TG accumulation, while induction of ERS stimulates hepatic lipogenesis. It was also demonstrated that these effects were partly mediated by ATF4. The results of the present study improve the understanding of the underlying pathophysiology of NAFLD and demonstrate the role of ATF4 in regulating lipogenesis and in the development of NAFLD. In addition, ATF4 may be considered a therapeutic target of NAFLD.

\section{Acknowledgements}

This study was supported by a grant from the National Natural Science Foundations of China (grant no. 81200639). The authors would like to thank Professor Jiming Ye (Health Innovations Research Institute, RMIT University, Melbourne, Victoria, Australia) for his advice on and interest in the study.

\section{References}

1. Malavolti M, Battistini NC, Miglioli L, Bagni I, Borelli L, Marino M, Scaglioni F and Bellentani S: Influence of lifestyle habits, nutritional status and insulin resistance in NAFLD. Front Biosci (Elite Ed) 4: 1015-1023, 2012.

2. Firneisz G: Non-alcoholic fatty liver disease and type 2 diabetes mellitus: The liver disease of our age? World J Gastroenterol 20: 9072-9089, 2014

3. Leite NC, Villela-Nogueira CA, Cardoso CR and Salles GF: Non-alcoholic fatty liver disease and diabetes: From physiopathological interplay to diagnosis and treatment. World J Gastroenterol 20: 8377-8392, 2014.

4. Perry RJ, Samuel VT, Petersen KF and Shulman GI: The role of hepatic lipids in hepatic insulin resistance and type 2 diabetes. Nature 510: 84-91, 2014.

5. Ren LP, Chan SM, Zeng XY, Laybutt DR, Iseli TJ, Sun RQ, Kraegen EW, Cooney GJ, Turner N and Ye JM: Differing endoplasmic reticulum stress response to excess lipogenesis versus lipid oversupply in relation to hepatic steatosis and insulin resistance. PLoS One 7: e30816, 2012.

6. Ozcan U, Yilmaz E, Ozcan L, Furuhashi M, Vaillancourt E, Smith RO, Görgün CZ and Hotamisligil GS: Chemical chaperones reduce ER stress and restore glucose homeostasis in a mouse model of type 2 diabetes. Science 313: 1137-1140, 2006.

7. Basseri S and Austin RC: Endoplasmic reticulum stress and lipid metabolism: Mechanisms and therapeutic potential. Biochem Res Int 2012: 841362, 2012.

8. Zhou H, Zhang K, Janciauskiene S and Li X: Endoplasmic reticulum stress and lipid metabolism. Biochem Res Int 2012: 257528, 2012

9. Volmer R and Ron D: Lipid-dependent regulation of the unfolded protein response. Curr Opin Cell Biol 33: 67-73, 2015.

10. Lee AH and Glimcher LH: Intersection of the unfolded protein response and hepatic lipid metabolism. Cell Mol Life Sci 66: 2835-2850, 2009.

11. Ozcan U, Cao Q, Yilmaz E, Lee AH, Iwakoshi NN, Ozdelen E, Tuncman G, Görgün C, Glimcher LH and Hotamisligil GS: Endoplasmic reticulum stress links obesity, insulin action and type 2 diabetes. Science 306: 457-461, 2004.

12. Ren LP, Song GY, Hu ZJ, Zhang M, Peng L, Chen SC, Wei L, Li F and Sun W: The chemical chaperon 4-phenylbutyric acid ameliorates hepatic steatosis through inhibition of de novo lipogenesis in high-fructose-fed rats. Int J Mol Med 32: 1029-1036, 2013.

13. Oyadomari S, Harding HP, Zhang Y, Oyadomari M and Ron D: Dephosphorylation of translation initiation factor 2alpha enhances glucose tolerance and attenuates hepatosteatosis in mice. Cell Metab 7: 520-532, 2008.

14. Wang C, Huang Z, Du Y, Cheng Y, Chen S and Guo F: ATF4 regulates lipid metabolism and thermogenesis. Cell Res 20: 174-184, 2010. 
15. Mohamed HA, Yao W, Fioravante D, Smolen PD and Byrne JH: cAMP-response elements in Aplysia creb1, creb2 and Ap-uch promoters: Implications for feedback loops modulating long term memory. J Biol Chem 280: 27035-27043, 2005.

16. Seo J, Fortuno ER III, Suh JM, Stenesen D, Tang W, Parks EJ, Adams CM, Townes T and Graff JM: Atf4 regulates obesity, glucose homeostasis and energy expenditure. Diabetes 58: 2565-2573, 2009.

17. Lange PS, Chavez JC, Pinto JT, Coppola G, Sun CW, Townes TM, Geschwind DH and Ratan RR: ATF4 is an oxidative stress-inducible, prodeath transcription factor in neurons in vitro and in vivo. J Exp Med 205: 1227-1242, 2008.

18. LiH, Meng Q, XiaoF, Chen S, Du Y, Yu J, Wang C and GuoF: ATF4 deficiency protects mice from high-carbohydrate-diet-induced liver steatosis. Biochem J 438: 283-289, 2011.

19. Xiao G, Zhang T, Yu S, Lee S, Calabuig-Navarro V, Yamauchi J, Ringquist $\mathrm{S}$ and Dong HH: ATF4 protein deficiency protects against high fructose-induced hypertriglyceridemia in mice.

20. Livak KJ and Schmittgen TD: Analysis of relative gene expression data using real-time quantitative PCR and the 2(-Delta Delta C(T)) Method. Methods 25: 402-408, 2001. J Biol Chem 288 25350-25361, 2013.

21. Kammoun HL, Chabanon H, Hainault I, Luquet S, Magnan C, Koike T, Ferré P and Foufelle F: GRP78 expression inhibits insulin and ER stress-induced SREBP-1c activation and reduces hepatic steatosis in mice. J Clin Invest 119: 1201-1215, 2009.

22. Zhang XQ, Xu CF, Yu CH, Chen WX and Li YM: Role of endoplasmic reticulum stress in the pathogenesis of nonalcoholic fatty liver disease. World J Gastroenterol 20: 1768-1776, 2014.

23. Thorburn AW, Storlien LH, Jenkins AB, Khouri S and Kraegen EW: Fructose-induced in vivo insulin resistance and elevated plasma triglyceride levels in rats. Am J Clin Nutr 49: 1155-1163, 1989.

24. Aragno M, Tomasinelli CE, Vercellinatto I, Catalano MG, Collino M, Fantozzi R, Danni O and Boccuzzi G: SREBP-1c in nonalcoholic fatty liver disease induced by Western-type high-fat diet plus fructose in rats. Free Radic Biol Med 47: 1067-1074, 2009.

25. Shimano H, Horton JD, Shimomura I, Hammer RE, Brown MS and Goldstein JL: Isoform 1c of sterol regulatory element binding protein is less active than isoform 1a in livers of transgenic mice and in cultured cells. J Clin Invest 99: 846-854, 1997.
26. Denechaud PD, Bossard P, Lobaccaro JM, Millatt L, Staels B, Girard J and Postic C: ChREBP, but not LXRs, is required for the induction of glucose-regulated genes in mouse liver. J Clin Invest 118: 956-964, 2008.

27. Malhi H and Kaufman RJ: Endoplasmic reticulum stress in liver disease. J Hepatol 54: 795-809, 2011.

28. Gentile CL, Frye $M$ and Pagliassotti MJ: Endoplasmic reticulum stress and the unfolded protein response in nonalcoholic fatty liver disease. Antioxid Redox Signal 15: 505-521, 2011.

29. Sha H, He Y, Chen H, Wang C, Zenno A, Shi H, Yang X, Zhang X and Qi L: The IRElalpha-XBP1 pathway of the unfolded protein response is required for adipogenesis. Cell Metab 9: 556-564, 2009.

30. Sriburi R, Jackowski S, Mori K and Brewer JW: XBP1: A link between the unfolded protein response, lipid biosynthesis and biogenesis of the endoplasmic reticulum. J Cell Biol 167: 35-41, 2004.

31. Lee AH, Scapa EF, Cohen DE and Glimcher LH: Regulation of hepatic lipogenesis by the transcription factor XBP1. Science 320: 1492-1496, 2008

32. Bobrovnikova-Marjon E, Hatzivassiliou G, Grigoriadou C, Romero M, Cavener DR, Thompson CB and Diehl JA: PERK-dependent regulation of lipogenesis during mouse mammary gland development and adipocyte differentiation. Proc Natl Acad Sci USA 105: 16314-16319, 2008.

33. Vallejo M, Ron D, Miller CP and Habener JF: C/ATF, a member of the activating transcription factor family of DNA-binding proteins, dimerizes with CAAT/enhancer-binding proteins and directs their binding to cAMP response elements. Proc Natl Acad Sci USA 90: 4679-4683, 1993.

34. Mamady H and Storey KB: Coping with the stress: Expression of ATF4, ATF6 and downstream targets in organs of hibernating ground squirrels. Arch Biochem Biophys 477: 77-85, 2008.

35. Wang $\mathrm{C}$ and Guo F: Effects of activating transcription factor 4 deficiency on carbohydrate and lipid metabolism in mammals. IUBMB Life 64: 226-230, 2012. 